\title{
Effect of Beryllium and Magnesium Doped Stanene Single Layer on Structural and Electronic Properties Using Density Functional Theory as Implemented in Quantum ESPRESSO
}

\author{
Alhassan Shuaibu1, Yakubu A Tanko1, Zainab Abdurrahman¹, Abdullahi Lawal', and \\ Maharaz M Nasir ${ }^{3}$ \\ ${ }^{1}$ Department of Physics, Faculty of Science, Kaduna State University, P.M.B, 2109, Kaduna, Nigeria. \\ ${ }^{2}$ Department of Physics, Federal College of Education Zaria, Kaduna, Nigeria. \\ ${ }^{3}$ Department of Physics, Federal University Duste, Jigawa State Nigeria. \\ Corresponding E-mail: alhazikara@gmail.com
}

Received 18-10-2020

Accepted for publication 03-03-2021

Published 15-03-2020

\begin{abstract}
Stanene is a quantum spin hall insulator and a favourable material for electronic and optoelectronic devices. Density functional theory (DFT) calculations are performed to study the band gap opening in stanene by investigating the effect of beryllium and magnesium doped stanene single layer to study the electronic and structural properties in stanene. The electronic band energy of pure stanene without spin orbit coupling (SOC) appear to show no energy gap at the Fermi level showing that stanene is a gapless material with Dirac cones at the $\mathrm{K}$ point and the band gap opens by a gap of $0.08 \mathrm{eV}$ is opened at the $\mathrm{K}$ point. The electronic structure of $\mathrm{Be}$ and $\mathrm{Mg}$ doped stanene shows that the Fermi level is shifted towards the valance band edge when compared to pure stanene. We have considered $6.25,12.5,18.75$ and $25 \%$ of both $\mathrm{Be}$ and $\mathrm{mg}$ doping. The electronic structure of Be doped stanene show that the Fermi level is shifted towards the valance band edge when compared to pure stanene. The Dirac point of stanene locates at $\Gamma$ shifted by 0.38 and $0.51 \mathrm{eV}$ for 6.25 and $12.5 \%$, an energy band gap of 0.27 and $0.50 \mathrm{eV}$ were obtained above the Fermi level for 6.25 and $12.5 \%$ respectively. In the case of $\mathrm{Mg}$, the bandgap remains slightly above the Fermi-level and amounts to $0.34 \mathrm{eV}$ for $6.25 \%$ and $0.43 \mathrm{eV}$ for $12.5 \%$, in the case of 18.75 and $25 \%$ the Dirac cone disappear completely, an energy gap of $0.28 \mathrm{eV}$ and $0.60 \mathrm{eV}$ were obtained above the Fermi level for 6.25 and $12.5 \%$ respectively, our findings show that the band gap of stanene open at $12.5 \%$ doping concentration of both $\mathrm{Be}$ and $\mathrm{Mg}$ impurities. These obtained band-gap value seem to be sufficient for use of alkaline earth metal doped stanene in optoelectronic and such applications where stanene is incapacitate for its use to switch on/off devices.
\end{abstract}

Keywords: Stanene; doped; Density functional theory; generalized gradient approximation; Quantum ESPRESSO 


\section{INTRODUCTION}

$\mathrm{F}$ ollowing the recent technological advancements produced by graphene because of its exceptional electronic and optical properties, there has been ongoing increasing interest in the research of two dimensional (2D) nanomaterials $[1,2]$. After, the synthesis of graphene, the heavy group-IV elements ( $\mathrm{Si}, \mathrm{Ge}$, and $\mathrm{Sn}$ ) are receiving considerable attention due to their similar electronic properties to that of graphene and broad application prospects that range from next generation energy efficient Nano-electronic devices [3 4 and 5]. For example, linear band dispersion at the Fermi energy, which leads to the charge carriers, behaves like massless Dirac Fermions [5,16 and 29]. These massless Dirac Fermions propagate with a very good Fermi velocity and show high carrier Mobility's at room temperature.

Nowadays, a new 2D group IV material, stanene, which is a buckled honeycomb structure of a 2D hexagonal tin film, has incited noteworthy interest due to its intriguing prospect as a quantum hall insulator, topological insulator and topological superconductor [6-8]. Stanene a bi-atomic layer of $\alpha$-Sn (111), which has a graphene-like honeycomb structure [9-10]. However, the structure is most stable with a buckled configuration due to weak $\pi-\pi$ bonding between Sn atoms [11]. Theoretical studies demonstrated that stanene has a zeroband gap without inclusion of spin orbit coupling (SOC), whereas it shows a band gap of $0.1 \mathrm{eV}$ after inclusion of SOC [12-13]. Stanene behaves like a quantum spin hall insulator (QSHI) and its derivatives show properties of large-gap quantum spin Hall insulators (QSHIs) [12]. Thus, such QSHIs show dissipation less conductor at room temperature. Furthermore, such QSHIs show excellent thermoelectric and quantum anomalous Hall (QAH) effect near room temperature [14]. On the other hand, doped QSHI materials show timereversal-invariant topological superconductivity. Thus, doped stanene could be a promising material for various applications [11]. On the other hand, the zero-band gap in stanene also limits its applications towards semiconductor based microelectronic devices [15-16]. Therefore, the expectation of a band gap opening in stanene has spurred intense scientific interests. Various approaches have been taken to modulate the electronic properties of stanene. Furthermore, the substrate plays an important role to tune the electronic properties of stanene due to the strong interaction [17 and 18]. Electronic properties of stanene are affected by the substrate because it induces band inversion in the bonding and antibonding states of stanene, which opens up the band gap in stanine [19]. Furthermore, van der Waals heterostructure of stanene modulates its electronic properties [20]. Recently, Khan et al. [21], demonstrated a heterostructure of stanene with h-BN sheet induces a tunable gap in stanine [21]. On the other hand, doping is also an efficient way to tune the electronic properties of $2 \mathrm{D}$ nanosheets by breaking the symmetry of the sublattice $[11-12,15]$. In this work, we have performed a First-Principles calculations of stanine doped group IV elements (Be and $\mathrm{Mg}$ ) to tune the electronic properties of stanene manuscript.

\section{COMPUTATIONAL DETAILS}

Our calculations were carried out within the density functional theory formalism (DFT) [22-23], as implemented in the Quantum ESPRESSO code [24]. We used the generalized gradient approximation (GGA) with the PerdewBurke-Ernzerhof (PBE) form to treat the exchange-correlation functional [25]. The norm-conserving Trouiller-Martins pseudopotential. In order to obtain the optimized configuration, the unit cells of considered Single layer were relaxed in axial direction until the stress components were less than $1 \mathrm{GPa}$. The conjugated-gradient optimization of atomic positions was allowed to proceed without any symmetry constraints until the forces acting on each atom were less than $0.02 \mathrm{eV} / \AA$. Also, the convergence criteria for total energy calculations was set at $10-4 \mathrm{eV}$. We used $50 \mathrm{k}$-points sampling according to Monkhorst-Pack approach for calculation of the total energy and band structure [26]. Also, XCrySDen (visualization for electronic and structural analysis) program was employed for visualization of the volumetric data such as electron/nuclear densities [27]. The Brillouin zone in our calculations is integrated with a $5 \times 5 \times$ 1 and $15 \times 15 \times 1$ Gamma-pack k-point grids for geometry optimization and density of state calculations, respectively. The vacuum space of $20 \AA$ is used in the z-direction to avoid any interaction between the adjacent repeating layers. Stanene possesses a low-buckled honeycomb structure, and this nonplanar geometry is the most stable structure. Stanene consists of tin atoms arranged in a single, hexagonal layer. The buckled geometry of stanene leads to the weakening of the $\pi-\pi$ bonding between tin atoms. This buckling and consequently bond weakening increase the overlap between $\pi$ and $\sigma$ orbitals, leading to the increase in the stability of the structure. $4 \times 4 \times$ 1 supercell of stanene was used for the electronic structure calculations. Fig. 1 displays the optimized structure of the pristine stanene supercell in different views. Beryllium $(\mathrm{Be})$ and Magnesium $(\mathrm{Mg})$ single atoms were substituted into the stanene single layer to model the doped stanene structures, and the effects of theses dopants on the structural and electronic properties were examined in detail. During the optimization of the structure, all atoms of the system were allowed to relax.

\section{RESULT AND DISCUSSION}

\section{A. Structural Stability}

Number The stability of stanene single layer usually achieved from its stable buckled geometry when compared with that of grapheme and germanene, this is because Stanene normally has a low-buckled non-planar honeycomb structure, and this non-planar geometry is the most stable structure. Stanene consists of tin atoms arranged in a single, hexagonal layer. The buckled geometry of stanene leads to the weakening 
of the $\pi-\pi$ bonding between tin atoms. This buckling and consequently bond weakening increases the overlap between $\pi$ and $\sigma$ orbitals, leading to the increase in the stability of the structure. In this work an initial four atoms hexagonal unit cell of statnene with space group P63/mmc (194) and crystal parameters of $\mathrm{a}=\mathrm{b}=462 \AA, \mathrm{c}=6.68 \AA$ has been increases and a $4 \times 4 \times 1$ supercell of 64 atoms was created by means of XCrysden software as shown in Fig. 1.

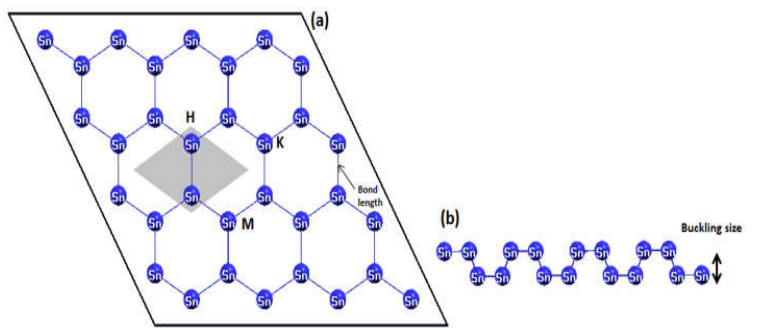

Fig. 1(a) The optimized $4 \times 4 \times 1$ supercell of stanene layer and (b) the buckling size.

After optimization by means of relaxation calculation within BFGS as implemented in Quantum ESPRESSO, we found that the optimized unit cell parameters and $\mathrm{Sn}-\mathrm{Sn}$ bond length are $\mathrm{a}=\mathrm{b}=4.67 \AA$ and $2.63 \AA$, respectively, this increase of 0.05 in the parameter may attribute due to the effect of spin orbit coupling (SOC) in the DFT pseudopontials. This result is in agreements with the number of DFT report [11-13].

In this work we adopt the model of substitutional doping along the reference point ( $\mathrm{H}, \mathrm{M}$ and $\mathrm{K}$ ) shown in Fig. 1 (a), our reference point was determined by chosen the one final energy as the most stable position. Table I and Fig. 2 shows the calculated total energy of the chosen points and optimized doped structures.

TABLE I CHOSEN DOPING REFERENCE POINT AND CALCULATED TOTAL ENERGY:

\begin{tabular}{lll}
\hline $\begin{array}{l}4 \times 4 \times 1 \text { supercell } \\
\text { of stanene }\end{array}$ & Total Energy (Ry) & Fermi energy $(\mathrm{eV})$ \\
\hline Along H-site & -2655.35966414 & 4.2178 \\
Along-M site & -2948.12311484 & 4.3210 \\
Along-K site & -2811.25381884 & 4.3379 \\
\hline
\end{tabular}

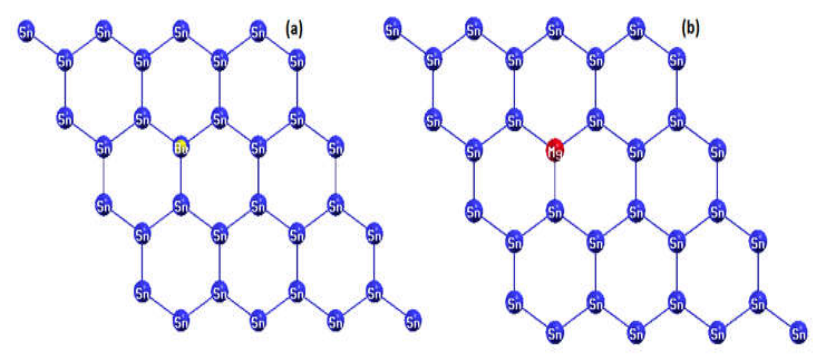

Fig. 2 (a) optimized Be-doped stanene (b) optimized Mg-doped stanene, all at reference point.
From Table I, H-site has been the chosen reference point for both the dopants (Be and $\mathrm{Mg}$ ). First of all, the formations energies with the two dopants $(\mathrm{Be}$ and $\mathrm{Mg}$ ) has been calculated in order to investigate which among them will be more stable using (1).

$E_{\mathrm{F}}=E_{\text {pure }}-\left(n_{\mathrm{Sn}} \mu_{\mathrm{Sn}}+\mu_{\text {dopant }}\right)$

where $E_{\mathrm{F}}$ is the formation energy, $E_{\text {pure }}$ is the total calculated energy of the pure stanene system $n_{\mathrm{Sn}}$ the number of $S n$ atoms in the unit cell, $\mu_{\mathrm{Sn}}$ is the chemical potential of $\mathrm{Sn}$ atom which is defined as energy per Sn atom in a perfect stanene and $\mu_{\text {dopant }}$ is the chemical potential of the dopants ( $\mathrm{Be}$ and $\mathrm{Mg}$ ) according to the energy per $\mathrm{Be}$ and $\mathrm{Mg}$ atom in Hexagonal $\mathrm{Be}$ and $\mathrm{Mg}$.

TABLE II CALCULATED FORMATION ENERGIES OF THE DOPED SYSTEMS:

\begin{tabular}{ll}
\hline Doped systems & Formation Energy $(\mathrm{eV})$ \\
\hline Be-Doped Stanene & 1.318 \\
Mg-Doped stanene & 1.425 \\
\hline
\end{tabular}

From Table II, the Mg-doped system has higher value than that of the Be-doped system, indicating that Be-doped system will be more stable than that of the Mg-doped, and this increase of positive formation energy may attribute due to the different in atomic radius of the dopants.

The doping concentrations has been investigated by varying the dopant as substituting one atom of Sn by one atom of the dopants $(\mathrm{Be}$ and $\mathrm{Mg}$ ) as $6.25 \%$, two atoms of Sn by two atoms of the dopants ( $\mathrm{Be}$ and $\mathrm{Mg}$ ) as $12.5 \%$, three $\mathrm{Sn}$ atoms by three atoms of the dopants ( $\mathrm{Be}$ and $\mathrm{Mg}$ ) as $18.75 \%$, and four $\mathrm{Sn}$ atoms by four atoms of the dopants (Be and $\mathrm{Mg}$ ) as $25 \%$, To investigate the structural distortion in the differently doped systems, the average bond lengths and total energy of the pure stanene and that of the differently doped systems were calculated and summarized in Table III.

TABLE III CALCULATED BOND LENGTHS OF THE DOPED SYSTEMS:

\begin{tabular}{|c|c|c|c|c|}
\hline \multirow{2}{*}{$\begin{array}{l}\text { Percentage } \\
\text { of Doped } \\
\text { systems }\end{array}$} & \multicolumn{2}{|c|}{ Bond length Sn-Sn $(\AA)$} & \multirow{2}{*}{$\begin{array}{l}\text { Bond } \\
\text { length } \\
\operatorname{Be}(\AA)\end{array}$} & \multirow{2}{*}{$\begin{array}{l}\text { Bond } \\
\text { length Sn- } \\
\mathrm{Mg}(\AA)\end{array}$} \\
\hline & Be-Doped & Mg-doped & & \\
\hline $6.25 \%$ & 2.5151 & 2.5382 & 1.9500 & 2.2300 \\
\hline $12.5 \%$ & 2.5021 & 2.5201 & 1.9959 & 2.4655 \\
\hline $18.75 \%$ & 2.5020 & 2.5200 & 1.9999 & 2.4768 \\
\hline $25 \%$ & 2.225 & 2.4112 & 2.1039 & 2.6331 \\
\hline
\end{tabular}

From Table III, a Single Be and $\mathrm{Mg}$ (6.25\%) doping at $\mathrm{Sn}$ reference site induces decreases in the $\mathrm{Sn}-\mathrm{Sn}$ bond lengths compared with that of the pure stanene single layer in both dopants with values $2.5151 \AA$ and $2.5382 \AA$, while $\mathrm{Sn}-\mathrm{Be}$ and Sn-Mg bond lengths become $1.95 \AA$ and $2.23 \AA$, respectively. This decrease in Sn-Sn bond lengths may be due to the difference in ionic radius between $\mathrm{Be}$ and $\mathrm{Mg}$, so substitution of $\mathrm{Be}$ and $\mathrm{Mg}$ at $\mathrm{Sn}$ site induces relatively small local structure 
modifications of such 2D materials this results is in consistent with experimental results [30]

On increasing the $\mathrm{Be}$ and $\mathrm{Mg}$ doping concentration to $12.5 \%$, Table III shows that the $\mathrm{Sn}-\mathrm{Sn}$ is $2.5021 \AA$ and $2.5201 \AA$, for the case of Be-doped and Mg-doped respectively showing more decreases as doping concentration increases, while $\mathrm{Sn}-\mathrm{Be}$ and $\mathrm{Sn}-\mathrm{Mg}$ bond length becomes $1.9959 \AA$ and $2.4655 \AA$ respectively, being greatly enlarged compared with the corresponding bond length of $6.25 \%$ doped stanene system. having $\mathrm{Be}$ and $\mathrm{Mg}$ doping concentration increases to $18.75 \%$, showed nearly the same bond length as that of $12.5 \%$, however the $\mathrm{Sn}-\mathrm{Be}$ and $\mathrm{Sn}-\mathrm{Mg}$ bond lengths was also slightly increases to $1.9999 \mathrm{~A}^{\circ}$ and $2.4768 \mathrm{~A}^{\circ}$. When four $\mathrm{Be}$ and $\mathrm{Mg}$ atoms were induced at the $\mathrm{Sn}$ sites of the stanene supercell, the doping concentration becomes $25 \%$ and the $\mathrm{Sn}-\mathrm{Sn}$ bond lengths became $2.225 \AA$ and $2.4112 \AA$ (showing the greatest shrinkage among all the doped system) while the Sn-Be, Sn$\mathrm{Mg}$ bond lengths became $2.1039 \AA$ and $2.6331 \AA$ showing a huge increase that making the layer of Stanene so distort.

\section{B. Electronic Properties}

The calculated electronic properties of pure stanene and stanene doped $\mathrm{Be}$ and $\mathrm{Mg}$ are computed within $\mathrm{PBE}$ approximation based on DFT along the high symmetry point of the first Brillouin zone (BZ). The calculated band structure of pure stanene are presented in Fig. 3 (a) and (b) show the graphs of band structure of stanene without and with spin orbit coupling (SOC). The electronic band energy of pure stanene without SOC appear to illustrate no energy gap at the Fermi level showing that stanene is a gapless material with Dirac cones at the $\mathrm{K}$ point and these findings are in good agreement with previous results $[11,28]$. Conversely, the band gap opens by including spin orbit coupling (SOC). Once SOC is considered, a gap of $0.08 \mathrm{eV}$ is opened at the $\mathrm{K}$ point, which is in good agreement with previous results [11,13].
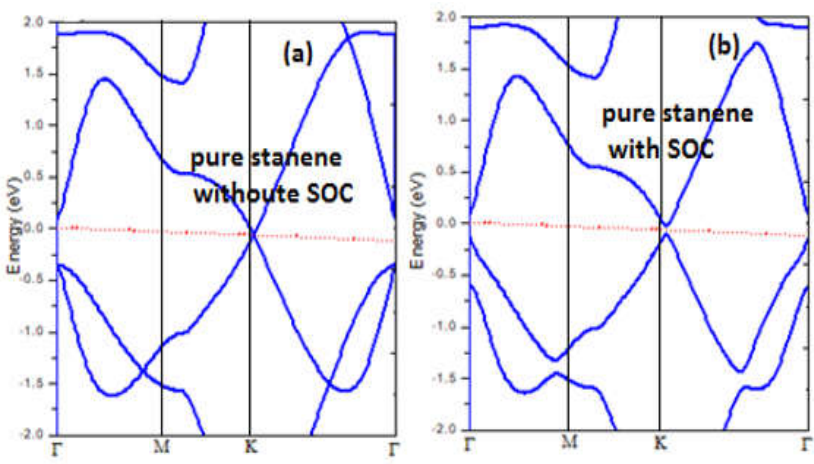

Fig. 3 Calculated Bands Energy of pure stanene: (a) without SOC (b) with SOC

The results of total density of states (DOS) and partial density of states (PDOS) of pure stanene are presented in Fig. 4 (a) and (b). The results of DOS help to further elaborate the nature of the band gap while PDOS gives information about the origin of bands. The orbital character reveals that the valence band maximum (VBM) and conduction band minimum
(CBM) are formed by $\mathrm{p}$ - and s-orbitals of $\mathrm{Sn}$ atoms. Furthermore, the s-orbital of $\mathrm{Sn}$ atoms contribute the most to the conduction bands than the p-orbital. Also, s-orbital of Sn atoms are predominantly to the valence bands near the Fermi level. Fig. 4 (b) shows that d-orbital of Sn has no contribution for both conduction bands and valence bands. Our results are in good agreement with previous first principles investigation [1,8 and 11].
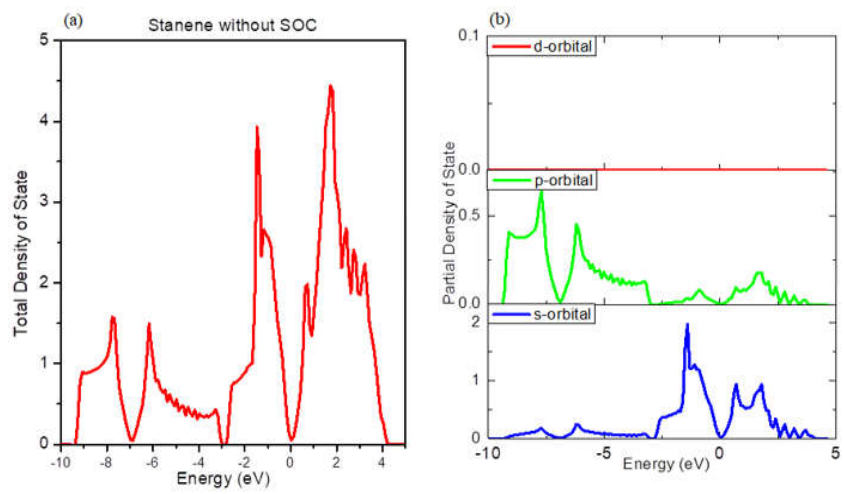

Fig. 4 (a) The calculated density of states of pure stanene (b) The calculated partial density of state (PDOS) of pure stanene.

\section{Doping}

One of the effective ways of tuning electronic properties of materials is substitutional elemental doping. Being stanene has similar electronic properties as graphene; band gap opening in stanene could be possible by elemental substitutional doping. The two atom of primitive unit cell of stanene is extended to $4 \times 4 \times 1$ supercell containing 64 atoms. $\mathrm{Sn}$ is a group IV element, and if we dope a Be and $\mathrm{Mg}$ then it induces a hole/electron in the system. Thus, it will break the symmetry of the sub lattice, which in turn, might change the electronic properties of the sheet.

\section{Stanene doped Be}

Beryllium $(\mathrm{Be})$ doped systems are widely investigated and found to be promising for electronic and related applications. The previous report demonstrated that introducing $\mathrm{Be}$ to graphene opens up its band gap, indicating that graphene doped $\mathrm{Be}$ is a semiconductor material with a band gap of 0.298 $\mathrm{eV}$ [22]. Here, we have considered $6.25 \%, 12.5 \%, 18.75 \%$ and $25 \%$ of Be doping i.e. one $\mathrm{Be}$ atom, two $\mathrm{Be}$ atoms three $\mathrm{Be}$ and four $\mathrm{Be}$ atoms is doped in the 64 atomic unit cell of stanene. Since higher doping concentration of Be modifies the structure of graphene [3], higher concentration of $\mathrm{Be}$ is also considered in order to modify the structure of stanene. The electronic band structure of Be doped stanene (see Fig. 5 (a) (d)) show that the Fermi level is shifted towards the valance band edge when compared to pure stanene and this confirms that the Be doped stanene is p-type of material. The Dirac point of stanene locates at $\Gamma$ shifted by 0.38 and $0.51 \mathrm{eV}$ for 6.25 and $12.5 \%$ respectively as shown in Fig. 5 (a) and (b). 
Interestingly, an energy band gaps of 0.27 and $0.50 \mathrm{eV}$ were obtained above the Fermi level for 6.25 and $12.5 \%$ respectively, which indicate that the Be doped stanene could be a degenerate semiconductor. Furthermore, in the case of $18.75 \%$ and $25 \%$ the Dirac cone vanished. Therefore, our findings show that the best doping concentration of $\mathrm{Be}$ is $12.5 \%$, because large energy gap was obtained at that percentage.
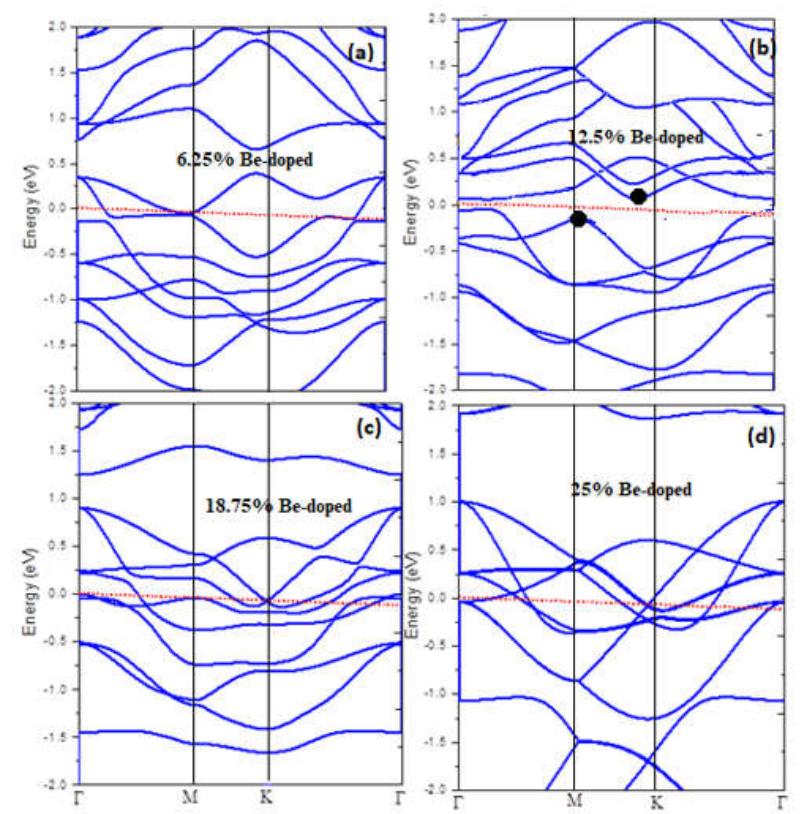

Fig. 5 Calculated Bands Energy of Stanene doped Be: (a) $6.25 \%$ (b) $12.5 \%$ (c) $18.75 \%$ and (d) $25 \%$

The DOS (Density of state) and PDOS (partial density of states) for the $\mathrm{Sn} \mathrm{Be}$ atoms of the Stanene doped $\mathrm{Be}$ are investigated through the GGA as illustrated in Fig. 6 (a) and (b). The DOS and PDOS plots in Fig. 5(b) clearly display that there is an energy range (below the Fermi, EF as well as above it). Furthermore, the DOS and PDOS plots in Fig. 6 reveal two main regions one in the $\mathrm{VB}$ and the other in the $\mathrm{CB}$. The first region below Fermi consists of the contributions by the s- and p-orbitals of $\mathrm{Sn}$ atoms and s-orbital of $\mathrm{Be}$ atoms. The conduction band region is mainly contributed by the p-orbitals of both $\mathrm{Sn}$ and $\mathrm{Be}$ atoms. It is also observed that the contribution of $\mathrm{p}$-orbital of $\mathrm{Be}$ is more than that of $\mathrm{Sn}$ to the conduction band while the s-orbital of $\mathrm{Sn}$ atoms are responsible for core bands.
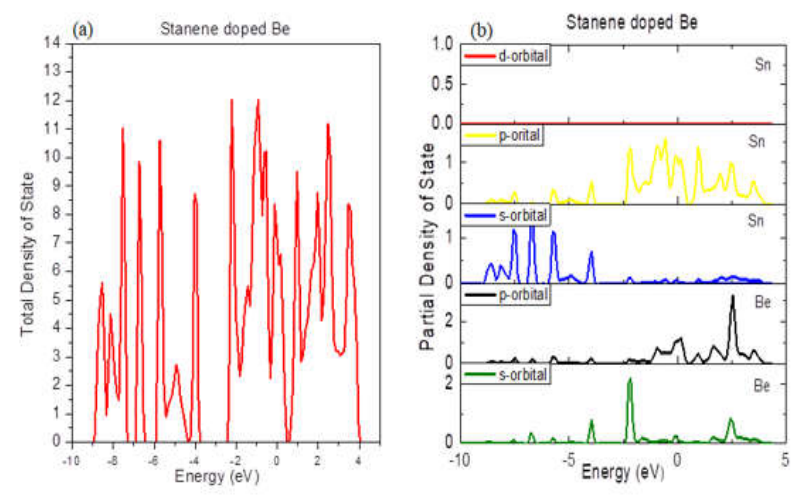

Fig. 6 Calculated DOS (Density of state) and (b) PDOS (partial density of states) for the Be atoms doped Stanene

\section{E. Stanene Doped $M g$}

The electronic properties of Stanene doped $\mathrm{Mg}$ in the hexagonal structure were executed based on PBE approximation. The band structure calculations are performed along special line of high symmetry points in the irreducible Brillouin zone $\Gamma\left(\begin{array}{lll}0 & 0 & 0\end{array}\right), \mathrm{M}\left(\begin{array}{lll}1 / 3 & 0 & 0\end{array}\right)$ and $\mathrm{K}\left(\begin{array}{lll}1 / 2 & 0 & 0\end{array}\right) \mathrm{k}$-space by setting Fermi level at $0 \mathrm{eV}$ on energy scale as shown in Fig. 6 , where the red dash line represents the Fermi level. The band structure of Stanene doped $\mathrm{Mg}$ was also computed for $6.25 \%$, $12.5 \%, 18.75 \%$ and $25 \%$ of $\mathrm{Mg}$ doping i.e. one $\mathrm{Mg}$ atom, two $\mathrm{Mg}$ atoms, three $\mathrm{Mg}$ atoms and four $\mathrm{Mg}$ atoms is doped in the 64 atomic supercell cells of stanene. The bandgap remains slightly above the Fermi-level and amounts to $0.34 \mathrm{eV}$ for 6.25 $\%$ and $0.43 \mathrm{eV}$ for $12.5 \%$, in the case of 18.75 and $25.0 \%$ the Dirac cone disappear completely. Conversely, an energy gap of $0.28 \mathrm{eV}$ and $0.60 \mathrm{eV}$ were obtained above the Fermi level for 6.25 and $12.5 \%$ respectively, showing that $\mathrm{Mg}$ doped stanene could be a degenerate semiconductor. Also, in the case of $18.75 \%$ and $25 \%$, the Dirac cone vanished. Our results reveal that a very high concentration of group II elements in stanene destroys the linear dispersion of $\pi$ bands (Dirac cones) at the Fermi level (Fig. 6 (c) and (d)). Therefore, our findings show that the best doping concentration of group II is $12.5 \%$, because large energy gap was obtained at that percentage. 

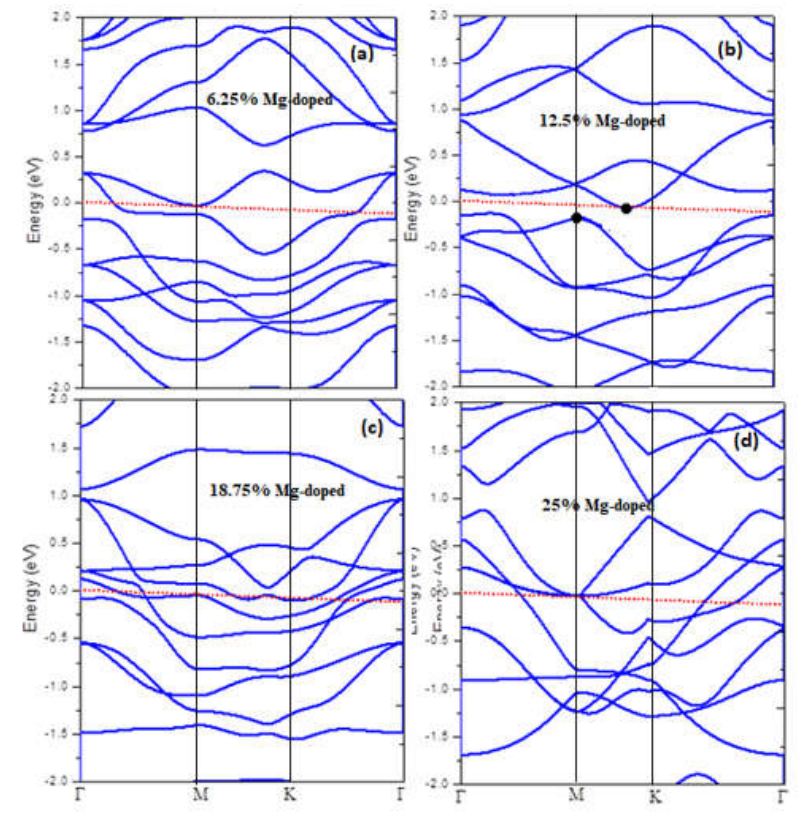

Fig. 7 Calculated Bands Energy of Stanene doped Mg: (a) $6.25 \%$ (b) $12.5 \%$ (c) $18.75 \%$ and (d) $25 \%$

We also have computed and analyzed the total density of states (TDOS) and partial density of states (PDOS) of stanene doped $\mathrm{Mg}$ as can be seen in Fig. 8. The result of DOS helps to elaborate the nature of the band gap and PDOS provides details information about the origin of bands for valence and conduction bands. The s-orbital of $\mathrm{Sn}$ atoms contribute to lowest valence band while middle of valence band which occurred approximately within $(0 \mathrm{eV}$ to $-5.0 \mathrm{eV})$ range is contributed from s-orbitals of $\mathrm{Mg}$ atoms and s-orbital of $\mathrm{Sn}$ atoms. In the unoccupied conduction bands above Fermi level $(0.0 \mathrm{eV}$ to $5.0 \mathrm{eV})$, the s-orbital of $\mathrm{Mg}$ atoms and p-orbitals of both $\mathrm{Sn}$ and $\mathrm{Mg}$ are the main contributors. In general, we noticed that p-orbital of $\mathrm{Sn}$ atoms are responsible for material properties near Fermi level.
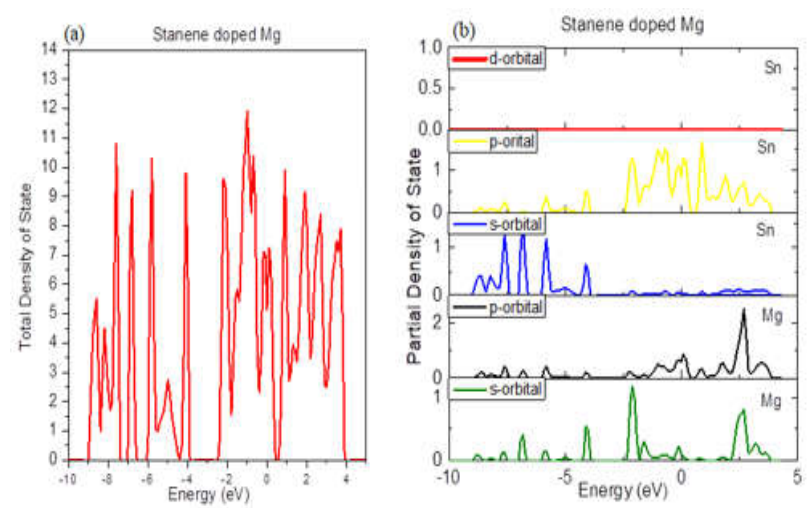

Fig. 8 Calculated DOS (Density of state) and (b) PDOS (partial density of states) for the $\mathrm{Mg}$ atoms doped Stanene

\section{CONCLUSION}

In summary we have investigated the electronic and structural properties of stanene single layer doped $\mathrm{Be}$ and $\mathrm{Mg}$ by means of DFT. Our findings show that the best doping concentration is $12.5 \%$ for both $\mathrm{Be}$ and $\mathrm{Mg}$ because, large energy gap was obtained at that percentage. The electronic structure of Be doped stanene shows that the Fermi level is shifted towards the valance band edge when compared to pure stanene and this confirms that the Be doped stanene is p-type of material. The Dirac point of stanene locates at $\Gamma$ shifted by 0.38 and $0.51 \mathrm{eV}$ for 6.25 and $12.5 \%$ respectively, an energy band gaps of 0.27 and $0.50 \mathrm{eV}$ were obtained above the Fermi level for 6.25 and $12.5 \%$ respectively, which indicate that the Be doped stanene could be a degenerate semiconductor. The band structure of Stanene doped $\mathrm{Mg}$ was also computed for $6.25,12.5,18.75$ and $25.0 \%$ of $\mathrm{Mg}$ doping i.e. $1 \mathrm{Mg}$ atom, $2 \mathrm{Mg}$ atoms, $3 \mathrm{Mg}$ atoms and $4 \mathrm{Mg}$ atoms is doped in the 64 atomic supercell cells of stanene. The band gap remains slightly above the Fermilevel and extents to $0.34 \mathrm{eV}$ for $6.25 \%$ and $0.43 \mathrm{eV}$ for 12.5 $\%$, in the case of 18.75 and $25.0 \%$ the Dirac cone disappear completely, an energy gap of $0.28 \mathrm{eV}$ and $0.60 \mathrm{eV}$ were obtained above the Fermi level for 6.25 and $12.5 \%$ Our results reveal that a very high concentration of alkaline earth elements in stanene destroys the linear dispersion of $\pi$ bands (Dirac cones) at the Fermi level. Thus finally, doping and manipulation of such impurities make stanene a reachable/ useful material and remove its disability for its employment in optoelectronics applications.

\section{References}

[1] V. Dhinakaran, M. Lavanya, K. Vigneswari, M. Ravichandran, and M. Vijayakumar, "Review on exploration of graphene in diverse applications and its future horizon". Materials Today: Proceedings. 2020.

[2] A. Manikandan, Y. -Z. Chen, C.-C. Shen, C. -W. Sher, H. -C. Kuo, and Y. -L. Chueh, "A critical review on two-dimensional quantum dots (2D QDs): From synthesis toward applications in energy and optoelectronics". Progress in Quantum Electronics, 100226. 2019.

[3] D. M Guzman, "Structural, Thermodynamic, and Electronic Properties of Mixed Ionic/Electronic Conductor Materials". Ph.D. Thesis. Purdue University, USA. 2018.

[4] F. Wang, Z. Wang, L. Yin, R. Cheng, J. Wang, Y. Wen and $X$. Zhan, "2D library beyond graphene and transition metal dichalcogenides: a focus on photodetection. Chemical Society Reviews, vol. 47, no. 16, pp. 6296-6341. 2018.

[5] X. Zhang, Z. Lai, Q. Ma, and H. Zhang, "Novel structured transition metal dichalcogenide nanosheets. Chemical Society Reviews, vol. 47, no. 9 , pp. 3301-3338. 2018.

[6] X. Chen, R. Meng, J. Jiang, Q. Liang, Q. Yang, C. Tan, T. Ren, "Electronic structure and optical properties of graphene/stanene heterobilayer". 
Physical Chemistry Chemical Physics, vol. 18, no. 24, pp. 16302-16309. 2016.

[7] Y. Shaidu and O. Akin-Ojo, "First principles predictions of superconductivity in doped stanene". Computational Materials Science, vol. 118, pp. 1115. 2016

[8] F. -f. Zhu, W. -j. Chen, Y. Xu, C. -1. Gao, D. -d. Guan, C. -h. Liu, J. -f. Jia, "Epitaxial growth of twodimensional stanene". Nature materials, vol. 14, no. 10, pp. 1020-1025. 2015.

[9] D. Di Sante, P. Eck, M. Bauernfeind, M. Will, R. Thomale, J. Schäfer, G. Sangiovanni, "Towards topological quasifreestanding stanene via substrate engineering”. Physical Review B, vol. 99, no. 3, pp. 035145. 2019.

[10] J. Falson, Y. Xu, M. Liao, Y. Zang, K. Zhu, C. Wang, K. He, "Type-II Ising Pairing in Few-Layer Stanene". arXiv preprint arXiv:1903.07627. 2019.

[11] P. Garg, I. Choudhuri and B. Pathak, "Stanene based gas sensors: effect of spin-orbit coupling". Physical Chemistry Chemical Physics, vol. 19, no. 46, pp. 31325-31334. 2017.

[12] P. Garg, G. Bhattacharyya, P. Bhauriyal, and B. Pathak, "Enhanced Lewis acid-base adducts in doped stanene: Sensing and photocatalysis". Applied Surface Science, vol. 478, pp. 946-958. 2019.

[13] P. Garg, I. Choudhuri, A. Mahata, and B. Pathak, "Band gap opening in stanene induced by patterned B-N doping". Physical Chemistry Chemical Physics, vol. 19, no. 5, pp. 3660-3669. 2017.

[14] W. Zhang, "Impact of Point Defects on Electronic Properties of Topological Materials". Rutgers The State University of New Jersey, School of Graduate Studies, 2019.

[15]A. Abbasi, "DFT study of the effects of AlP pair doping on the structural and electronic properties of stanene nanosheets. Physica E: Low-dimensional Systems and Nanostructures, vol. 108, pp. 34-43. 2019.

[16]A. Abbasi, J. J. Sardroodi, "Electronic structure tuning of stanene monolayers from DFT calculations: Effects of substitutional elemental doping". Applied Surface Science, vol. 456, pp. 290301. 2018.

[17]X. Chen, R. Meng, J. Jiang, Q. Liang, Q. Yang, C. Tan and T. Ren, "Electronic structure and optical properties of graphene/stanene heterobilayer". Physical Chemistry Chemical Physics, vol. 18, no. 24, pp. 16302-16309. 2016

[18] T. S. Hartman and Z. K. Sofer, "Beyond Graphene: Chemistry of Group 14 Graphene Analogues: Silicene, Germanene, and Stanene. ACS nano, vol. 13, no. 8, pp. 8566-8576. 2019.

[19] N. Ferdous, M. S. Islam, J. Park, and A. Hashimoto, "Tunable electronic properties in stanene and two dimensional silicon-carbide heterobilayer: A first principles investigation". AIP Advances, vol. 9, no. 2, 025120. 2019
[20] A. S., Rashid, M. S. Islam, N. Ferdous, K. N. Anindya, J. Park, and A. Hashimoto, "Widely tunable electronic properties in graphene/twodimensional silicon carbide van der Waals heterostructures". Journal of Computational Electronics, vol. 18, no. 3, pp. 836-845. 2019.

[21]K. Khan, A. K. Tareen, M. Aslam, R. Wang, Y. Zhang, A. Mahmood, and Z Guo, "Recent developments in emerging two-dimensional materials and their applications. Journal of Materials Chemistry C. 2020.

[22] R. G. Parr, "Density functional theory of atoms and molecules". In Horizons of quantum chemistry, pp. 5-15. Springer, Dordrecht. 1980.

[23]L. J. Sham, and M. Schlüter, "Density-functional theory of the energy gap. Physical Review Letters, vol. 51, no. 20, 1888. 1983.

[24]P. Giannozzi, et al., "QUANTUM ESPRESSO: a modular and open-source software project for quantum simulations of materials. Journal of physics: Condensed matter, vol. 21, no. 39, 395502. 2009.

[25] Z. Wu, and R. E. Cohen, "More accurate generalized gradient approximation for solids. Physical Review $B$, vol. 73 , no. 23, 235116. 2006.

[26]H. J. Monkhorst and J. D. Pack, "Special points for Brillouin-zone integrations. Physical review B, vol. 13, no. 12, pp. 5188. 1976.

[27]A. Kokalj, "XCrySDen-a new program for displaying crystalline structures and electron densities. Journal of Molecular Graphics and Modelling, vol. 17, no. 3-4, pp. 176-179. 1999.

[28]M. Ghadiyali and S. Chacko, "Band splitting in bilayer stanene electronic structure scrutinized via first principle DFT calculations. Computational Condensed Matter, vol. 17, e00341. 2018.

[29] J. Shim, E. K. Lee, Y. J. Lee, and R. M. Nieminen, "Density-functional calculations of defect formation energies using supercell methods: Defects in diamond". Physical Review B, vol. 71, no. 3, 035206. 2005.

[30]C. Chen, J. Zhang, B. Zhang and H. Ming Duan, "Hydrogen adsorption of Mg-doped graphene oxide: a first-principles study". The Journal of Physical Chemistry C, vol. 117, no. 9, pp. 4337-4344. 2013. 